\title{
In reply to the letter to the editor concerning "Reliability of diagnostic imaging for degenerative diseases with osseous changes in the temporomandibular joint with special emphasis on subchondral cyst"
}

\author{
Michihito Nozawa ${ }^{1} \cdot$ Nobumi Ogi $^{2} \cdot$ Yoshiko Ariji $^{1} \cdot$ Yoshitaka Kise $^{1} \cdot$ Miwa Nakayama ${ }^{1} \cdot$ Masako Nishiyama $^{1}$. \\ Munetaka Naitoh ${ }^{1} \cdot$ Kenichi Kurita $^{2} \cdot$ Eiichiro Ariji $^{1}$
}

Published online: 27 May 2020

(c) Japanese Society for Oral and Maxillofacial Radiology and Springer Nature Singapore Pte Ltd. 2020

Thank you for the opportunity to reply the letter regarding our manuscript entitled "Reliability of diagnostic imaging for degenerative diseases with osseous changes in the temporomandibular joint with special emphasis on subchondral cysts". We also thank the author of the letter for attention to our manuscript. Our reply is as follows:

Thank you for your useful comments on our manuscript. As you mentioned, the kappa value depends on the prevalence of each category. However, it is impossible to know the prevalence before experiments because of missing of gold standards in this kind of study. Probably the prevalence would follow the natural prevalence in TMD patients. Initially, we had considered the use of Fleiss's methodology for many observers [1]. Although it could clarify the overall consistency, the tendency of each observer could not be found. When one of three observers had a different assessment policy for osseous changes from other two observers, the consistencies of two pairs would show low values. We wanted to know such differences in osseous change assessments. As a result, the kappa values were not so different among all three pairs for almost categories. However, we have also applied the Fleiss's method to our data resulting in almost values equivalent to those in the present study. For example, the overall consistencies are 0.61 and 0.65 for the panoramic TMJ projection and CT, respectively. In addition to the Fleiss's method, we had initially tried to apply the Landis's method for categorical data [2].

Michihito Nozawa

illehan@dpc.agu.ac.jp

1 Department of Oral and Maxillofacial Radiology, AichiGakuin University School of Dentistry, 2-11 Suemori-dori, Chikusa-ku, Nagoya 464-8651, Japan

2 Department of Oral and Maxillofacial Surgery, Aichi-Gakuin University School of Dentistry, 2-11 Suemori-dori, Chikusa-ku, Nagoya 464-8651, Japan
However, in the evaluation, we could not always select one of five categories, erosion, osteophyte, subchondral cyst and generalized sclerosis together with non-osseous change, for one condyle. Two or more changes simultaneously occurred in one condyle. Moreover, each category could not be ordered according to its severity. Therefore, the Landis's method and weighted kappa statics were considered not to be appropriate, and we separately evaluated the consistency in the presence or absence of each category of osseous change.

We believe that the results, namely, the location and the rate of cortical layer presence in the cyst-like area on CT, support the bone contusion theory. However, the description of "A possible improvement in reliability is suggested relative to MRI findings" was somewhat confused to understand. Your suggestions of "Any conclusion in agreement needs to be supported by the methodological and statistical issues mentioned above" is completely correct. In this sentence, we wanted to emphasize the clearness of MR appearances and they would be easy to interpret for subchondral cyst. So, when the evaluation of subchondral cyst could be performed in relation to MR findings, the reliability would be improved. This did not mean that the CT or panoramic radiography showed higher reliability than MR.

Thank you again for your useful comments that help us in the future study.

\section{Reference}

1. Fleiss JL. Measuring nominal scale agreement among many raters. Psychol Bull. 1971;76:378-82.

2. Landis R, Koch GG. The measurement of observer agreement for categorical data. Biometrics. 1977;33:150-74.

Publisher's Note Springer Nature remains neutral with regard to jurisdictional claims in published maps and institutional affiliations. 